\title{
Observational constraints on Barrow holographic dark energy
}

\author{
Fotios K. Anagnostopoulos ${ }^{1, a}$, Spyros Basilakos ${ }^{2}$, Emmanuel N. Saridakis ${ }^{3,4,5, b}$ \\ ${ }^{1}$ Department of Physics, National and Kapodistrian University of Athens, Zografou Campus GR, 15773 Athens, Greece \\ ${ }^{2}$ Research Center for Astronomy and Applied Mathematics, Academy of Athens, Soranou Efesiou 4, 11527 Athens, Greece \\ ${ }^{3}$ National Observatory of Athens, Lofos Nymfon, 11852 Athens, Greece \\ ${ }^{4}$ Department of Physics, National Technical University of Athens, Zografou Campus GR, 15773 Athens, Greece \\ 5 Department of Astronomy, School of Physical Sciences, University of Science and Technology of China, Hefei 230026, \\ People's Republic of China
}

Received: 28 July 2020 / Accepted: 14 August 2020 / Published online: 5 September 2020

(C) The Author(s) 2020

\begin{abstract}
We use observational data from Supernovae (SNIa) Pantheon sample, as well as from direct measurements of the Hubble parameter from the cosmic chronometers (CC) sample, in order to extract constraints on the scenario of Barrow holographic dark energy. The latter is a holographic dark energy model based on the recently proposed Barrow entropy, which arises from the modification of the black-hole surface due to quantum-gravitational effects. We first consider the case where the new deformation exponent $\Delta$ is the sole model parameter, and we show that although the standard value $\Delta=0$, which corresponds to zero deformation, lies within the $1 \sigma$ region, a deviation is favored. In the case where we let both $\Delta$ and the second model parameter to be free we find that a deviation from standard holographic dark energy is preferred. Additionally, applying the Akaike, Bayesian and Deviance Information Criteria, we conclude that the one-parameter model is statistically compatible with $\Lambda \mathrm{CDM}$ paradigm, and preferred comparing to the two-parameter one. Finally, concerning the present value of the Hubble parameter we find that it is close to the Planck value.
\end{abstract}

\section{Contents}

1 Introduction . . . . . . . . . . . . . . 1

2 Barrow holographic dark energy . . . . . . . . . . 2

3 Data and methodology . . . . . . . . . . . . 3

3.1 Cosmological probes . . . . . . . . . . 3

3.1 .1 Type Ia Supernovae . . . . . . . . . . . . 3

3.1.2 Cosmic chronometers . . . . . . . . . 3

3.1 .3 Joint analysis . . . . . . . . . . . 3

\footnotetext{
a e-mail: svasil@ academyofathens.gr

b e-mail: msaridak@phys.uoa.gr (corresponding author)
}

3.2 Information criteria and model selection . . . . 4

4 Observational constraints ........... 4

5 Conclusions .............. 7

References . . . . . . . . . . . . . . 7

\section{Introduction}

Accumulated data from various probes lead to the safe deduction that the universe have undergone two phases of accelerated expansion, at early and late cosmological times respectively. Such a behavior may require the introduction of extra degrees of freedom that are capable of triggering it (the simple cosmological constant can sufficiently describe the latter phase, but it is not adequate to describe the former one). A first main direction is the construct modified gravitational theories, that posses general relativity as a particular limit, but which on larger scales can produce the above phenomenology, such as in $f(R)$ gravity [1-3], $f(G)$ gravity [4], Galileon theory [5], $f(T)$ gravity [6-8], Finsler gravity [9] etc (see [10-13] for reviews). The second main direction is to maintain general relativity as the underlying gravitational theory and introduce the the inflaton field(s) $[14,15]$ and/or the dark energy concept attributed to new fields, particles or fluids $[16,17]$.

One interesting approach for the description of dark energy arises from holographic considerations [18-22]. Specifically, since the largest length of a quantum field theory is connected to its Ultraviolet cutoff [23], one can result to a vacuum energy which at cosmological scales forms a form of holographic dark energy [24,25]. Holographic dark energy is very efficient in quantitatively describe the late-time acceleration [24-35] and it is in agreement with observational data [36-44]. Hence, many extensions of the basic scenario have appeared in the literature, based mainly on the use of differ- 
ent horizons as the largest distance (i.e. the universe "radius") [45-70].

One such extension is Barrow holographic dark energy, which arises by applying the usual holographic principle but using the recently proposed Barrow entropy instead of the Bekenstein-Hawking one. The later is a modification of the black-hole entropy caused by quantum-gravitational effects that deform the horizon, leading it to acquire a fractal, intricate, structure [71]. Hence, one results with an extended holographic dark energy, which includes basic holographic dark energy as a sub-case in the limit where Barrow entropy becomes the Bekenstein-Hawking one, but which in general is a novel scenario which exhibits more interesting and richer phenomenology [72].

In the present work we desire to use observational data from from Supernovae (SNIa) Pantheon sample, and from direct Hubble constant measurements with cosmic chronometers (CC), in order to constrain Barrow holographic dark energy, and in particular to impose observational bounds in the new Barrow exponent that quantifies the quantumgravitational deformation and thus the deviation from usual holographic dark energy. The plan of the work is the following: in Sect. 2 we briefly review Barrow holographic dark energy. In Sect. 3 we present the various datasets, the applied methodology, and the information criteria that we will use. In Sect. 4 we provide the obtained results and we give the corresponding contour plots. Finally, in Sect. 5 we summarize and conclude.

\section{Barrow holographic dark energy}

In this section we present the cosmological scenario of Barrow holographic dark energy (for other cosmological applications of Barrow entropy see $[73,74]$ ). Barrow entropy is a quantum-gravitationally corrected black-hole entropy due to the fractal structure brought about in its horizon, and it takes the form [71]

$S_{B}=\left(\frac{A}{A_{0}}\right)^{1+\Delta / 2}$,

where $A$ is the standard horizon area and $A_{0}$ the Planck area. The quantum deformation, and hence the deviation from Bekenstein-Hawking entropy is quantified by the new exponent $\Delta$, which takes the value $\Delta=0$ in the standard, nondeformed case, while for $\Delta=1$ it corresponds to maximal deformation.

We consider a flat Friedmann-Robertson-Walker (FRW) geometry with metric

$d s^{2}=-d t^{2}+a^{2}(t) \delta_{i j} d x^{i} d x^{j}$, where $a(t)$ is the scale factor. As it was shown in [72], application of the holographic principle but using Barrow entropy (2.1), leads to Barrow holographic dark energy, whose energy density reads:

$\rho_{D E}=C R_{h}^{\Delta-2}$

where $C$ is a parameter with dimensions $[L]^{-2-\Delta}$, and $R_{h}$ the future event horizon

$R_{h} \equiv a \int_{t}^{\infty} \frac{d t}{a}=a \int_{a}^{\infty} \frac{d a}{H a^{2}}$,

where $H \equiv \dot{a} / a$ is the Hubble parameter.

The two Friedmann equations are

$3 M_{p}^{2} H^{2}=\rho_{m}+\rho_{D E}$

$-2 M_{p}^{2} \dot{H}=\rho_{m}+p_{m}+\rho_{D E}+p_{D E}$,

with $M_{p}=1 / \sqrt{8 \pi G}$ the Planck mass. Moreover, $p_{D E}$ is the pressure of Barrow holographic dark energy, and $\rho_{m}$, $p_{m}$ are respectively the energy density and pressure of the matter fluid. As usual we consider the two sector to be noninteracting, and thus the usual conservation equations hold

$$
\begin{aligned}
& \dot{\rho}_{m}+3 H\left(\rho_{m}+p_{m}\right)=0, \\
& \dot{\rho}_{D E}+3 H \rho_{D E}\left(1+w_{D E}\right)=0 .
\end{aligned}
$$

In the following we focus on the case of dust matter, namely we assume that $p_{m}=0$.

Introducing the density parameters $\Omega_{i} \equiv \frac{1}{3 M_{p}^{2} H^{2}} \rho_{i}$, in the case $0 \leq \Delta<1$ one can easily extract the evolution equation for $\Omega_{D E}$ as a function of $x \equiv \ln a=-\ln (1+z)$, with $z$ the redshift (with $a_{0}=1$ ), namely [72]

$$
\begin{gathered}
\frac{\Omega_{D E}^{\prime}}{\Omega_{D E}\left(1-\Omega_{D E}\right)}=\Delta+1+Q\left(1-\Omega_{D E}\right)^{\frac{\Delta}{2(\Delta-2)}} \\
\left(\Omega_{D E}\right)^{\frac{1}{2-\Delta}} e^{\frac{3 \Delta}{2(\Delta-2)} x},
\end{gathered}
$$

with

$Q \equiv(2-\Delta)\left(\frac{C}{3 M_{p}^{2}}\right)^{\frac{1}{\Delta-2}}\left(H_{0} \sqrt{\Omega_{m 0}}\right)^{\frac{\Delta}{2-\Delta}}$

a dimensionless parameter and where primes denote derivatives with respect to $x$. Furthermore, the equation of state for Barrow holographic dark energy, i.e $w_{D E} \equiv p_{D E} / \rho_{D E}$, is given by

$$
w_{D E}=-\frac{1+\Delta}{3}-\frac{Q}{3}\left(\Omega_{D E}\right)^{\frac{1}{2-\Delta}}\left(1-\Omega_{D E}\right)^{\frac{\Delta}{2(\Delta-2)}} e^{\frac{3 \Delta}{2(2-\Delta)} x}
$$


Barrow holographic dark energy is a new dark energy scenario. In the case $\Delta=0$ it coincides with standard holographic dark energy $\rho_{D E}=3 c^{2} M_{p}^{2} R_{h}^{-2}$, with $C=3 c^{2} M_{p}^{2}$ the model parameter. In this case (2.9) becomes $\left.\Omega_{D E}^{\prime}\right|_{\Delta=0}=$ $\Omega_{D E}\left(1-\Omega_{D E}\right)\left(1+2 \sqrt{\frac{3 M_{p}^{2} \Omega_{D E}}{C}}\right)$, and can be analytically solved implicitly [24], while $\left.w_{D E}\right|_{\Delta=0}=-\frac{1}{3}-\frac{2}{3} \sqrt{\frac{3 M_{p}^{2} \Omega_{D E}}{C}}$, which is the standard holographic dark energy result [25]. However, in the case $\Delta>0$, where the deformation effects switch on, the scenario at hand departs from the standard one, leading to different cosmological behavior. Lastly, in the upper limit $\Delta=1$, it coincides with $\Lambda \mathrm{CDM}$ cosmology.

\section{Data and methodology}

In this section we provide the various data sets that are going to be used for the observational analysis, and then we present the statistical methods that we employ. We use data from Supernovae type Ia observations together with direct $H(z)$ Hubble data, and we apply the method of maximum likelihood analysis to in order to extract constraints on the free model parameters. As a final step, we will employ known information criteria in order to assess the quality of the fittings.

\subsection{Cosmological probes}

\subsubsection{Type Ia Supernovae}

Perhaps the most known and frequently used cosmological probe are distant Type Ia Supernovae. A supernova explosion is an extremely luminous event, with its brightness being comparable with the brightness of its host galaxy [75]. The observed light curves posses peak brightness mostly unaffected by the distance, thus can be used as standard candles. Specifically, one could use the observed distance modulo, $\mu_{o b s}$, to constrain cosmological models. We use the most recent data set available, namely the binned Pantheon dataset described at [75]. Finally, the corresponding likelihood reads

$\mathcal{L}_{\text {SNia }}(Y ; \mathcal{M}) \sim \exp \left(-\frac{1}{2} \sum_{i=1}^{40} m_{i} C_{c o v}^{-1} m_{i}^{\dagger}\right)$,

where $\mathrm{Y}$ is the vector of the free parameters of the cosmological model, $m_{i}=\mu_{\text {obs }, i}-\mu_{\text {theor }}\left(z_{i}\right)-\mathcal{M}$ and $\mu_{\text {theor }}=$ $5 \log \left(\frac{D_{L}}{1 M p c}\right)+25$, and $D_{L}$ is the standard luminocity distance, given as $D_{L}=c(1+z) \int_{0}^{z} \frac{1}{H(z)}$, that holds for a flat FRWL space-time, regardless of the underlying cosmology. Finally, $C_{c o v}$ is the covariance matrix of the binned Pantheon dataset. The parameter $\mathcal{M}$ is an intrinsic free parameter to the Pantheon dataset and quantifies a variety of observational uncertainties, i.e host galaxy properties, etc.

\subsubsection{Cosmic chronometers}

Data from the so-called "cosmic chronometers" (CC), are measurements of the Hubble rate, based upon the estimation of the differential age of passive evolving galaxies. The latter are galaxies with their emission spectra dominated by old stars population. The central idea is to use the definition of the Hubble rate, re-parametrized in terms of redshift, i.e

$H \equiv \frac{\dot{a}}{a}=-\frac{1}{1+z} \frac{d z}{d t}$.

From this point, the redshift is relatively easily observed spectroscopically and the remaining work is to estimate the quantity $d z / d t$. As it was firstly proposed by Jimenez and Loeb in [76], this is possible via measuring the age difference between two sets of passively evolving galaxies, lying within a small redshift difference. The observational method and specific information from an astrophysical point of view are described in detail in $[77,78]$.

From a cosmological viewpoint, it is important to note that data from cosmic chronometers are essentially model independent, as long as we work within an FRWL spacetime without extrinsic curvature. Furthermore, the redshift range of the available cosmic chronometers extends to 2, thus they allow for more stringent constraints to the cosmological models under study. Thus, cosmic chronometers are used widely in the field [42,79-81]. In this work the sub-sample of [82], consisting of only CC data, is employed. The likelihood for the cosmic chronometers, assuming gaussian errors, reads

$\mathcal{L}_{C C}(Y) \sim \exp \left[-\frac{1}{2} \sum_{i=0}^{31} \frac{\left(H\left(z_{i}\right)_{\text {theor }}-H_{\text {obs }, i}\right)^{2}}{\sigma_{i}^{2}}\right]$,

where $\sigma_{i}$ are the corresponding errors.

\subsubsection{Joint analysis}

In order to obtain the joint observational constraints on the cosmological scenario by using $P$ cosmological datasets, we first introduce the total likelihood function as

$\mathcal{L}_{\text {tot }}(Y)=\prod_{p=1}^{P} \mathcal{L}_{i}$,

assuming Gaussian errors, and where no correlation between various data sets employed. Hence, the total $\chi_{\text {tot }}^{2}$ function will 
be

$\chi_{\mathrm{tot}}^{2}=\sum_{p=1}^{P} \chi_{P}^{2}$.

The parameter vector has dimension $k$, namely the $v$ parameters of the scenario, plus the number of hyper-parameters $v_{\text {hyp }}$ of the applied datasets, i.e. $k=v+v_{\text {hyp }}$. For the scenario of Barrow holographic dark energy, and since we are using Hublle rate and SNIa data, the free parameters are contained in the vector $a_{m}=\left(\Omega_{m 0}, C, \Delta, h, \mathcal{M}\right)$, with $h=H_{0} / 100$. We apply the Markov Chain Monte Carlo (MCMC) algorithm in the environment of the Python package emcee [83], and we perform the minimization of $\chi^{2}$ with respect to $a_{m}$. We use 800 chains (walkers) and 3500 steps (states). Lastly, the convergence of the algorithm is verified using auto-correlation time considerations, and additionally we employ the Gelman-Rubin criterion [84] too for completeness.

\subsection{Information criteria and model selection}

As a final step, we apply the known Akaike Information Criterion (AIC) [85] and the Bayesian Information Criterion (BIC) [86], and the Deviance Information Criterion [87], in order to examine the quality of the fittings and hence the relevant observational compatibility of the scenarios.

The AIC is based on information theory, and it is an estimator of the Kullback-Leibler information with the property of asymptotically unbiasedness. Under the standard assumption of Gaussian errors, the corresponding estimator reads as $[88,89]$

$\mathrm{AIC}=-2 \ln \left(\mathcal{L}_{\text {max }}\right)+2 k+\frac{2 k(k+1)}{N_{\text {tot }}-k-1}$,

with $\mathcal{L}_{\text {max }}$ the maximum likelihood of the datasets and $N_{\text {tot }}$ the total data points. For large number of data points $N_{\text {tot }}$ it reduces to $\mathrm{AIC} \simeq-2 \ln \left(\mathcal{L}_{\max }\right)+2 k$. On the other hand, the $\mathrm{BIC}$ criterion is an estimator of the Bayesian evidence [88-90], given by

BIC $=-2 \ln \left(\mathcal{L}_{\max }\right)+k \log \left(N_{\text {tot }}\right)$.

Finally, the DIC criterion is based on concepts from both Bayesian statistics and information theory [87], and it is written as [90]

$\mathrm{DIC}=D\left(\overline{a_{m}}\right)+2 C_{B}$.

The variable $C_{B}$ is the Bayesian complexity given as $C_{B}=$ $\overline{D\left(a_{m}\right)}-D\left(\overline{a_{m}}\right)$, with overlines denoting the standard mean value. Moreover, $D\left(a_{m}\right)$ is the Bayesian Deviation, a quantity closely related to the effective degrees of freedom [87], which for the general class of exponential distributions, it reads as $D\left(a_{m}\right)=-2 \ln \left(\mathcal{L}\left(a_{m}\right)\right)$.

In order to compare a set of $n$ models we utilize the above criteria by extracting the relative difference of the involved IC values $\Delta \mathrm{IC}_{\text {model }}=\mathrm{IC}_{\text {model }}-\mathrm{IC}_{\text {min }}$, where $\mathrm{IC}_{\min }$ is the minimum IC value in the set of compared models [91]. We then assign a "probability of correctness" to each model using the rule $[88,89]$

$P \simeq \frac{e^{-\Delta \mathrm{IC}_{i}}}{\sum_{i=1}^{n} e^{-\Delta \mathrm{IC}_{i}}}$,

with $i$ running over the set of $n$ models. The quantity $P$ can be considered as a measure for the relative strength of observational support between these two models. In particular, employing the Jeffreys scale $[92,93]$, the condition $\Delta \mathrm{IC} \leq 2$ implies statistical compatibility of the model at hand with the reference model, the condition $2<\Delta \mathrm{IC}<6$ corresponds to a middle tension between the two models, while $\Delta \mathrm{IC} \geq 10$ implies a strong tension.

\section{Observational constraints}

In this section we confront the scenario of Barrow holographic dark energy with cosmological data from Supernovae type Ia observations as well as from direct measurements of the Hubble rate, i.e. $H(z)$ data, under the procedure described above. We are interested in extracting the constraints on the basic model parameter $\Delta$, which quantifies the deviation from standard entropy, as well as on the secondary parameter $C$. We start by performing the analysis keeping $C$ fixed to the value $C=3$ in $M_{p}^{2}$ units, that is to the value for which Barrow holographic dark energy restores exactly standard holographic dark energy in the limit $\Delta=0$. In this case we can investigate purely the effect and the implications of the Barrow exponent $\Delta$. Additionally, as a next step we perform the full fitting procedure, handling both $\Delta$ and $C$ as free parameters.

In Table 1 we summarize the results for the parameters. Moreover, in Figs. 1 and 2 we present the corresponding likelihood contours. In the case where $C$ is kept fixed, we observe that $\Delta=0.095_{-0.100}^{+0.093}$. As we can see, the standard value $\Delta=0$ is inside the $1 \sigma$ region, however the mean value is $\Delta=0.095$ and thus a deviation from the standard case is preferred. Furthermore, we can see that $h=0.6895_{-0.0189}^{+0.0187}$ i.e we obtain an $H_{0}$ value close to the Planck one $H_{0}=$ $67.37 \pm 0.54 \mathrm{~km} \mathrm{~s}^{-1} \mathrm{Mpc}^{-1}$ [94] instead to the direct value $H_{0}=74.03 \pm 1.42 \mathrm{~km} \mathrm{~s}^{-1} \mathrm{Mpc}^{-1}$ [95], which was somehow expected since the Hubble parameter is constrained only from the $\mathrm{CC}$ data, since the distance modulus from supernovae Ia cannot directly constrain $H_{0}$. 
Table 1 Observational constraints on the parameters of Barrow holographic dark energy (BHDE), and the corresponding $\mathcal{L}_{\text {max }}$, using SN Ia and CC datasets

\begin{tabular}{|c|c|c|c|c|c|c|}
\hline Models & $\Omega_{m 0}$ & $h$ & $C$ & $\Delta$ & $\mathcal{M}$ & $2 \ln \mathcal{L}_{\text {max }}$ \\
\hline$\left.\mathrm{BHDE}\right|_{\text {Cfixed }}$ & $0.285_{-0.044}^{+0.043}$ & $0.6895_{-0.0189}^{+0.0187}$ & 3 & $0.095_{-0.100}^{+0.093}$ & $-19.390_{-0.055}^{+0.056}$ & 53.843 \\
\hline$\left.\mathrm{BHDE}\right|_{\text {Cfree }}$ & $0.284_{-0.044}^{+0.043}$ & $0.6892_{-0.0189}^{+0.0187}$ & $3.421_{-1.611}^{+1.753}$ & $0.094_{-0.101}^{+0.094}$ & $-19.390_{-0.056}^{+0.055}$ & 53.978 \\
\hline$\Lambda C D M$ & $0.300_{-0.021}^{+0.022}$ & $0.6907_{-0.0196}^{+0.0200}$ & - & - & $-16.996_{-0.059}^{+0.057}$ & 54.003 \\
\hline
\end{tabular}
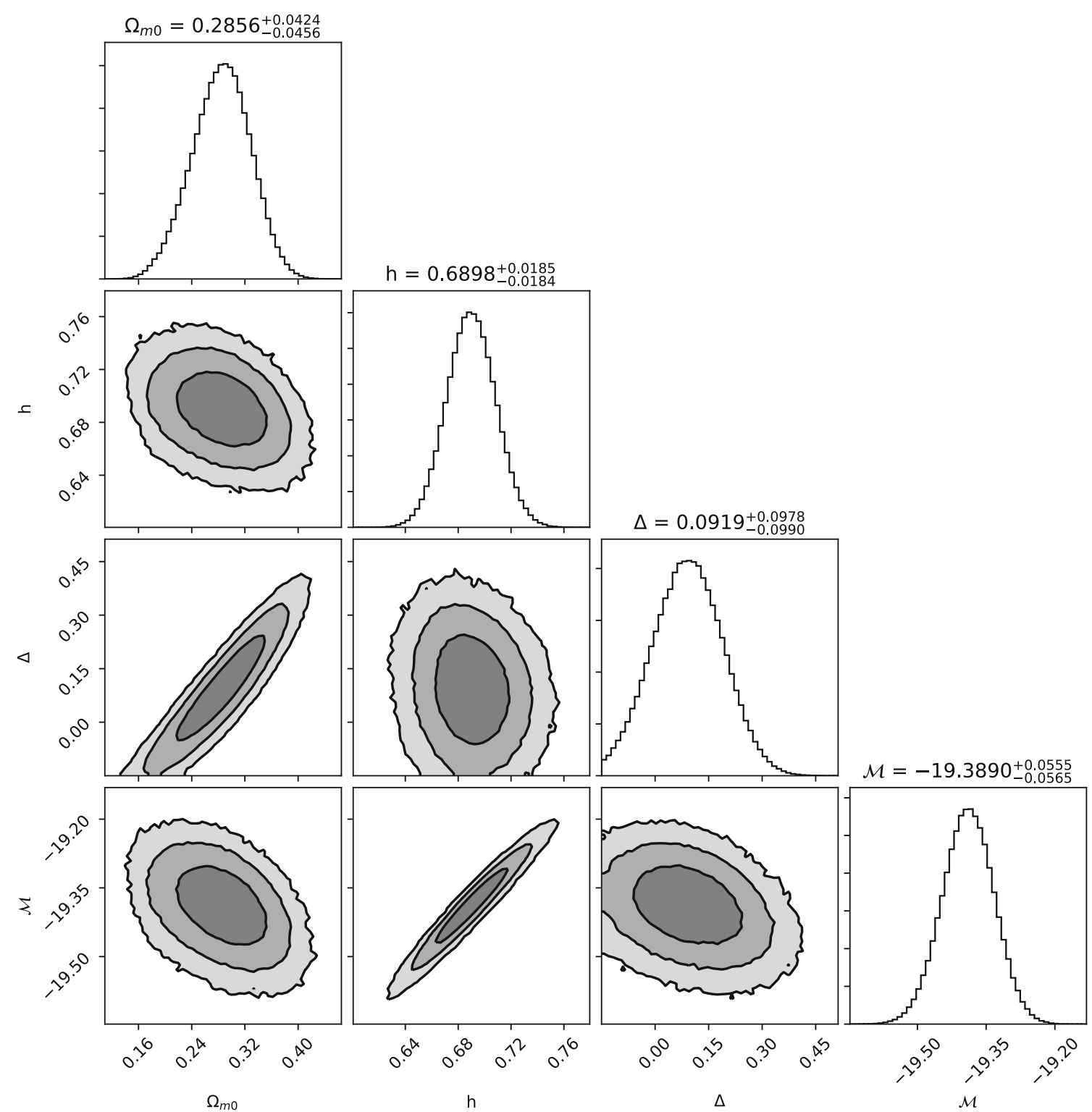

$\Delta$

$\mathcal{M}$

Fig. 1 The $1 \sigma, 2 \sigma$ and $3 \sigma$ likelihood contours for Barrow holographic dark energy, in the case where we fix the model parameter $C=3$ in $M_{p}$ units, using SNIa and $H(z)$ data. Additionally, we present the involved 1-dimensional (1D) marginalized posterior distributions and

In the case where both $\Delta$ and $C$ are free parameters, we observe that $\Delta=0.094_{-0.101}^{+0.093}$, which is quite similar with the previous $C$-fixed case. This implies that the the parameters mean values corresponding to the $1 \sigma$ area of the MCMC chain. $\mathcal{M}$ is the usual free parameter of SNIa data that quantifies possible astrophysical systematic errors, [75]. For these fittings we obtain $\chi_{\min }^{2} / d o f=0.8031$

deformation exponent $\Delta$ is constrained not to have its standard value, i.e. deviation from standard holographic dark energy is slightly favored. Concerning the parameter $C$ we 


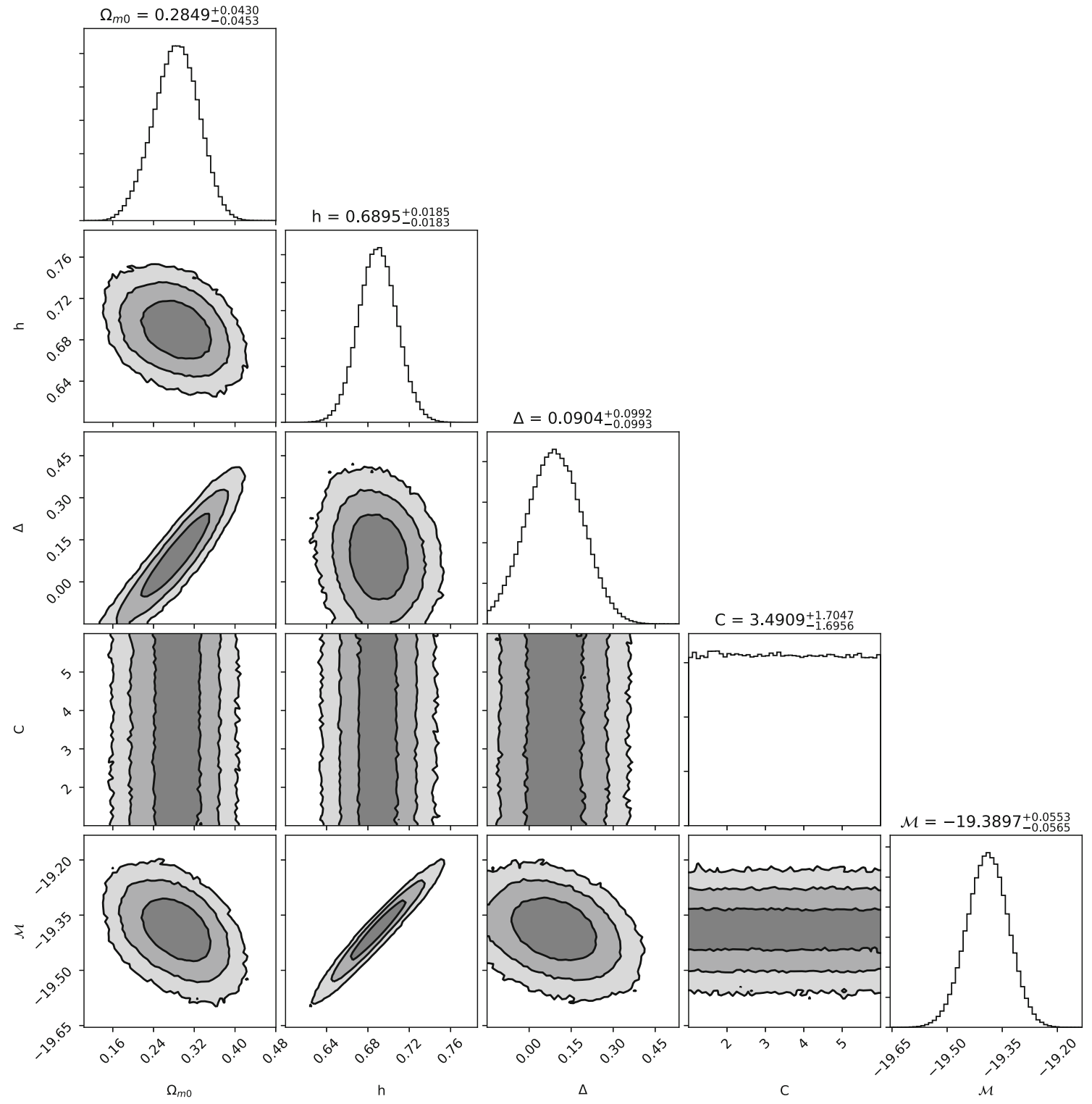

Fig. 2 The $1 \sigma, 2 \sigma$ and $3 \sigma$ likelihood contours for Barrow holographic dark energy, in the case where both $\Delta$ and $C$ are free parameters, using SNIa and $H(z)$ data. Additionally, we present the involved 1dimensional (1D) marginalized posterior distributions and the parame- ters mean values corresponding to the $1 \sigma$ area of the MCMC chain. $\mathcal{M}$ is the usual free parameter of SNIa data that quantifies possible astrophysical systematic errors [75]. For these fittings we obtain $\chi_{\min }^{2} /$ dof $=0.8179$

free scenario, as the extra free parameter does not contribute in the fit. This becomes evident from Fig. 2, where the $1 \sigma$ area of the parameter $C$ is not closed. Due to the latter fact, the DIC criterion cannot quantify well the adequacy of the $C$-free model. Thus, it is imperative to use AIC to proceed with model selection. However, to compare the other two models, one can still use DIC. As $\triangle D I C$ is smaller than 2, $C$-fixed and $\Lambda \mathrm{CDM}$ are statistically equivalent. Using AIC to compare all models used here, we find that $C$-free model is in middle tension with $\Lambda \mathrm{CDM}$ while $C$-fixed is statistically 
Table 2 The information criteria AIC, BIC and DIC for the examined cosmological models, along with the corresponding differences $\triangle \mathrm{IC} \equiv$ $\mathrm{IC}-\mathrm{IC}_{\min }$

\begin{tabular}{lllllll}
\hline Model & AIC & $\Delta$ AIC & BIC & $\Delta$ BIC & DIC & $\Delta$ DIC \\
\hline${\text { BHDE }\left.\right|_{\text {Cfixed }}}$ & 62.449 & 2.088 & 70.894 & 4.103 & 61.591 & 1.683 \\
BHDE $_{\text {Cfree }}$ & 64.901 & 4.540 & 75.292 & 8.501 & 61.118 & 1.210 \\
$\Lambda \mathrm{CDM}$ & 60.361 & 0 & 66.791 & 0 & 59.908 & 0 \\
\hline
\end{tabular}

equivalent with $\Lambda \mathrm{CDM}$. Finally, $\Lambda \mathrm{CDM}$ paradigm seems to be slightly statistically preferred.

\section{Conclusions}

In this work used observational data from Supernovae (SNIa) Pantheon sample, as well as from direct measurements of the Hubble parameter from the cosmic chronometers (CC) sample, in order to extract constraints on the scenario of Barrow holographic dark energy. The latter is a new holographic dark energy scenario which is based on the recently proposed Barrow entropy, which arises from the modification of the black-hole surface due to quantum-gravitational effects. In particular, the deformation from standard BekensteinHawking entropy is quantified by the new exponent $\Delta$, with $\Delta=0$ corresponding to standard case, while $\Delta=1$ to maximal deformation. Hence, for $\Delta=0$ Barrow holographic dark energy coincides with standard holographic dark energy, while for $0<\Delta<1$ it corresponds to a new cosmological scenario that proves to lead to interesting and rich behavior [72]. Lastly, in the limiting case $\Delta=1$ one obtains $\rho_{D E}=$ const.$=\Lambda$ and hence $\Lambda \mathrm{CDM}$ paradigm is restored, through a a completely different physical framework.

We first considered the case where the new exponent $\Delta$ is the sole model parameter, in order to investigate its pure effects, i.e. we fixed the model parameter $C$ to its value for which Barrow holographic dark energy restores exactly standard holographic dark energy in the limit $\Delta=0$. As we showed, the standard value $\Delta=0$ is inside the $1 \sigma$ region, however the mean value is $\Delta=0.094$, namely a deviation is favored. Additionally, for the Hubble rate we obtained a value $0.6895_{-0.0189}^{+0.0187}$ close to the Planck instead to the direct value, which was expected since the Hubble parameter is constrained only from the CC data, since the distance modulus from supernovae Ia cannot directly constrain $H_{0}$.

In the case where we let both $\Delta$ and $C$ to be free model parameters, we found that $0.094_{-0.101}^{+0.094}$, and hence deviation from standard holographic dark energy is preferred. Concerning the Hubble rate we found that it is close to the Planck value too.

Finally, we performed a comparison of Barrow holographic dark energy with the concordance $\Lambda \mathrm{CDM}$ paradigm, using the AIC, BIC and DIC information criteria. As we showed, the one-parameter scenario is statistically compatible with $\Lambda \mathrm{CDM}$, and preferred comparing to the twoparameter one. In summary, Barrow holographic dark energy is in agreement with cosmological data, and it can serve as a good candidate for the description of nature.

Data Availability Statement This manuscript has no associated data or the data will not be deposited. [Authors' comment: All data that have been used in our analysis have already been freely released and have been published by the corresponding research teams. In our text we properly give all necessary References to these works, and hence no further data deposit is needed.]

Open Access This article is licensed under a Creative Commons Attribution 4.0 International License, which permits use, sharing, adaptation, distribution and reproduction in any medium or format, as long as you give appropriate credit to the original author(s) and the source, provide a link to the Creative Commons licence, and indicate if changes were made. The images or other third party material in this article are included in the article's Creative Commons licence, unless indicated otherwise in a credit line to the material. If material is not included in the article's Creative Commons licence and your intended use is not permitted by statutory regulation or exceeds the permitted use, you will need to obtain permission directly from the copyright holder. To view a copy of this licence, visit http://creativecomm ons.org/licenses/by/4.0/.

Funded by $\mathrm{SCOAP}^{3}$.

\section{References}

1. A.A. Starobinsky, A new type of isotropic cosmological models without singularity. Phys. Lett. B 91, 99 (1980)

2. A. De Felice, S. Tsujikawa, $\mathrm{f}(\mathrm{R})$ theories. Living Rev. Relativ. 13, 3 (2010). arXiv: 1002.4928

3. S. Nojiri, S.D. Odintsov, Unified cosmic history in modified gravity: from $\mathrm{F}(\mathrm{R})$ theory to Lorentz non-invariant models. Phys. Rep. 505, 59 (2011). arXiv: 1011.0544

4. S. Nojiri, S.D. Odintsov, Modified Gauss-Bonnet theory as gravitational alternative for dark energy. Phys. Lett. B 631, 1 (2005). arXiv:hep-th/0508049

5. C. Deffayet, G. Esposito-Farese, A. Vikman, Covariant Galileon. Phys. Rev. D 79, 084003 (2009). arXiv:0901.1314

6. G.R. Bengochea, R. Ferraro, Dark torsion as the cosmic speed-up. Phys. Rev. D 79, 124019 (2009). arXiv:0812.1205

7. S.H. Chen, J.B. Dent, S. Dutta, E.N. Saridakis, Cosmological perturbations in $\mathrm{f}(\mathrm{T})$ gravity. Phys. Rev. D 83, 023508 (2011). arXiv: 1008.1250

8. G. Kofinas, E.N. Saridakis, Teleparallel equivalent of GaussBonnet gravity and its modifications. Phys. Rev. D 90, 084044 (2014)

9. S. Basilakos, A.P. Kouretsis, E.N. Saridakis, P. Stavrinos, Resembling dark energy and modified gravity with Finsler-Randers cosmology. Phys. Rev. D 88, 123510 (2013). arXiv:1311.5915

10. S. Nojiri, S.D. Odintsov, Introduction to modified gravity and gravitational alternative for dark energy. eConf C 0602061, 06 (2006). arXiv:hep-th/0601213

11. S. Nojiri, S.D. Odintsov, Introduction to modified gravity and gravitational alternative for dark energy. Int. J. Geom. Meth. Mod. Phys. 4, 115 (2007). arXiv:hep-th/0601213

12. S. Capozziello, M. De Laurentis, Extended theories of gravity. Phys. Rep. 509, 167 (2011). arXiv:1108.6266 
13. Y.F. Cai, S. Capozziello, M. De Laurentis, E.N. Saridakis, f(T) teleparallel gravity and cosmology. Rep. Prog. Phys. 79, 106901 (2016). arXiv:1511.07586

14. K.A. Olive, Inflation. Phys. Rep. 190, 307 (1990)

15. N. Bartolo, E. Komatsu, S. Matarrese, A. Riotto, Non-Gaussianity from inflation: theory and observations. Phys. Rep. 402, 103 (2004). arXiv:astro-ph/0406398

16. E.J. Copeland, M. Sami, S. Tsujikawa, Dynamics of dark energy. Int. J. Mod. Phys. D 15, 1753 (2006). arXiv:hep-th/0603057

17. Y.F. Cai, E.N. Saridakis, M.R. Setare, j-Q Xia, Quintom cosmology: theoretical implications and observations. Phys. Rep. 493, 1 (2010). arXiv:0909.2776

18. G. 't Hooft, Dimensional reduction in quantum gravity. Salamfest 1993, 0284-296. arXiv:gr-qc/9310026

19. L. Susskind, The world as a hologram. J. Math. Phys. 36, 6377 (1995). arXiv:hep-th/9409089

20. R. Bousso, The holographic principle. Rev. Mod. Phys. 74, 825 (2002). arXiv:hep-th/0203101

21. W. Fischler, L. Susskind, Holography and cosmology. arXiv:hep-th/9806039

22. P. Horava, D. Minic, Probable values of the cosmological constant in a holographic theory. Phys. Rev. Lett. 85, 1610 (2000). arXiv:hep-th/0001145

23. A.G. Cohen, D.B. Kaplan, A.E. Nelson, Effective field theory, black holes, and the cosmological constant. Phys. Rev. Lett. 82, 4971 (1999). arXiv:hep-th/9803132

24. M. Li, A model of holographic dark energy. Phys. Lett. B 603, 1 (2004). arXiv:hep-th/0403127

25. S. Wang, Y. Wang, M. Li, Holographic dark energy. Phys. Rep. 696, 1 (2017). arXiv:1612.00345

26. R. Horvat, Holography and variable cosmological constant. Phys. Rev. 70, 087301 (2004). arXiv:astro-ph/0404204

27. Q.G. Huang, M. Li, The Holographic dark energy in a non-flat universe. JCAP 0408, 013 (2004). arXiv:astro-ph/0404229

28. D. Pavon, W. Zimdahl, Holographic dark energy and cosmic coincidence. Phys. Lett. B 628, 206 (2005). arXiv:gr-qc/0505020

29. B. Wang, Y.G. Gong, E. Abdalla, Transition of the dark energy equation of state in an interacting holographic dark energy model. Phys. Lett. B 624, 141 (2005). arXiv:hep-th/0506069

30. S. Nojiri, S.D. Odintsov, Unifying phantom inflation with latetime acceleration: scalar phantom-non-phantom transition model and generalized holographic dark energy. Gen. Relativ. Grav. 38, 1285 (2006). arXiv:hep-th/0506212

31. H. Kim, H.W. Lee, Y.S. Myung, Equation of state for an interacting holographic dark energy model. Phys. Lett. B 632, 605 (2006). arXiv:gr-qc/0509040

32. B. Wang, C.Y. Lin, E. Abdalla, Constraints on the interacting holographic dark energy model. Phys. Lett. B 637, 357 (2006). arXiv:hep-th/0509107

33. M.R. Setare, E.N. Saridakis, Interacting holographic dark energy model in non-flat universe. Phys. Lett. B 642, 1 (2006). arXiv:hep-th/0609069

34. M.R. Setare, E.N. Saridakis, Non-minimally coupled canonical, phantom and quintom models of holographic dark energy. Phys. Lett. B 671, 331 (2009). arXiv:0810.0645

35. M.R. Setare, E.N. Saridakis, Correspondence between Holographic and Gauss-Bonnet dark energy models. Phys. Lett. B 670, 1 (2008). arXiv:0810.3296

36. X. Zhang, F.Q. Wu, Constraints on holographic dark energy from Type Ia supernova observations. Phys. Rev. D 72, 043524 (2005). arXiv:astro-ph/0506310

37. M. Li, X.D. Li, S. Wang, X. Zhang, Holographic dark energy models: a comparison from the latest observational data. JCAP 0906 , 036 (2009). arXiv:0904.0928

38. C. Feng, B. Wang, Y. Gong, R.K. Su, Testing the viability of the interacting holographic dark energy model by using combined observational constraints. JCAP 0709, 005 (2007). arXiv:0706.4033

39. X. Zhang, Holographic Ricci dark energy: current observational constraints, quintom feature, and the reconstruction of scalar-field dark energy. Phys. Rev. D 79, 103509 (2009). arXiv:0901.2262

40. J. Lu, E.N. Saridakis, M.R. Setare, L. Xu, Observational constraints on holographic dark energy with varying gravitational constant. JCAP 1003, 031 (2010). arXiv:0912.0923

41. S.M.R. Micheletti, Observational constraints on holographic tachyonic dark energy in interaction with dark matter. JCAP 1005, 009 (2010). arXiv:0912.3992

42. R. D'Agostino, Holographic dark energy from nonadditive entropy: cosmological perturbations and observational constraints. Phys. Rev. D 99(10), 103524 (2019). arXiv:1903.03836

43. E. Sadri, Observational constraints on interacting Tsallis holographic dark energy model Eur. Phys. J. C 79(9), 762 (2019). arXiv:1905.11210

44. Z. Molavi, A. Khodam-Mohammadi, Observational tests of GaussBonnet like dark energy model. Eur. Phys. J. Plus 134(6), 254 (2019). arXiv:1906.05668

45. Y.G. Gong, Extended holographic dark energy. Phys. Rev. D 70, 064029 (2004). arXiv:hep-th/0404030

46. E.N. Saridakis, Restoring holographic dark energy in brane cosmology. Phys. Lett. B 660, 138 (2008). arXiv:0712.2228

47. M.R. Setare, E.C. Vagenas, The Cosmological dynamics of interacting holographic dark energy model. Int. J. Mod. Phys. D 18, 147 (2009). arXiv:0704.2070

48. R.G. Cai, A Dark Energy Model Characterized by the Age of the Universe. Phys. Lett. B 657, 228 (2007). arXiv:0707.4049

49. E.N. Saridakis, Holographic dark energy in Braneworld models with moving Branes and the $\mathrm{w}=-1$ crossing. JCAP 0804, 020 (2008). arXiv:0712.2672

50. E.N. Saridakis, Holographic dark energy in Braneworld models with a Gauss-Bonnet term in the bulk. Interacting behavior and the $\mathrm{w}=-1$ crossing. Phys. Lett. B 661, 335 (2008). arXiv:0712.3806

51. M.R. Setare, E.C. Vagenas, Thermodynamical interpretation of the interacting holographic dark energy model in a non-flat universe. Phys. Lett. B 666, 111 (2008). arXiv:0801.4478

52. M. Jamil, E.N. Saridakis, M.R. Setare, Holographic dark energy with varying gravitational constant. Phys. Lett. B 679, 172 (2009). arXiv:0906.2847

53. Y. Gong, T. LI, A modified holographic dark energy model with infrared infinite extra dimension(s). Phys. Lett. B 683, 241 (2010). arXiv:0907.0860

54. M. Suwa, T. Nihei, Observational constraints on the interacting Ricci dark energy model. Phys. Rev. D 81, 023519 (2010). arXiv:0911.4810

55. M. Bouhmadi-Lopez, A. Errahmani, T. Ouali, The cosmology of an holographic induced gravity model with curvature effects. Phys. Rev. D 84, 083508 (2011). arXiv:1104.1181

56. L.P. Chimento, M.G. Richarte, Interacting dark matter and modified holographic Ricci dark energy induce a relaxed Chaplygin gas. Phys. Rev. D 84, 123507 (2011). arXiv:1107.4816

57. M. Malekjani, Generalized holographic dark energy model described at the Hubble length Astrophys. Space Sci. 347, 405 (2013). arXiv: 1209.5512

58. L.P. Chimento, M. Forte, M.G. Richarte, Modified holographic Ricci dark energy coupled to interacting dark matter and a non interacting baryonic component. Eur. Phys. J. C 73(1), 2285 (2013). arXiv:1301.2737

59. M. Khurshudyan, J. Sadeghi, R. Myrzakulov, A. Pasqua, H. Farahani, Interacting quintessence dark energy models in Lyra manifold. Adv. High Energy Phys. 2014, 878092 (2014). arXiv:1404.2141

60. R.C.G. Landim, Holographic dark energy from minimal supergravity. Int. J. Mod. Phys. D 25(4), 1650050 (2016). arXiv:1508.07248 
61. A. Pasqua, S. Chattopadhyay, R. Myrzakulov, Power-law entropycorrected holographic dark energy in Hoava-Lifshitz cosmology with Granda-Oliveros cut-off. Eur. Phys. J. Plus 131(11), 408 (2016). arXiv: 1511.00611

62. A. Jawad, N. Azhar, S. Rani, Entropy corrected holographic dark energy models in modified gravity. Int. J. Mod. Phys. D 26(4), 1750040 (2016)

63. B. Pourhassan, A. Bonilla, M. Faizal, E.M.C. Abreu, Holographic dark energy from fluid/gravity duality constraint by cosmological observations. Phys. Dark Univ. 20, 41 (2018). arXiv:1704.03281

64. S. Nojiri, S.D. Odintsov, Covariant generalized holographic dark energy and accelerating universe. Eur. Phys. J. C 77(8), 528 (2017). arXiv: 1703.06372

65. E.N. Saridakis, Ricci-Gauss-Bonnet holographic dark energy. Phys. Rev. D 97(6), 064035 (2018). arXiv:1707.09331

66. E.N. Saridakis, K. Bamba, R. Myrzakulov, F.K. Anagnostopoulos, Holographic dark energy through Tsallis entropy. JCAP 12, 012 (2018). arXiv:1806.01301

67. y Aditya, S. Mandal, P. Sahoo, D. Reddy, Observational constraint on interacting Tsallis holographic dark energy in logarithmic Brans-Dicke theory. Eur. Phys. J. C 79(12), 1020 (2019). arXiv: 1910.12456

68. S. Nojiri, S.D. Odintsov, E.N. Saridakis, Holographic inflation. Phys. Lett. B 797, 134829 (2019). arXiv:1904.01345

69. C.Q. Geng, Y.T. Hsu, J.R. Lu, L. Yin, Modified cosmology models from thermodynamical approach. Eur. Phys. J. C 80(1), 21 (2020). arXiv: 1911.06046

70. S. Waheed, Econstruction paradigm in a class of extended teleparallel theories using Tsallis holographic dark energy. Eur. Phys. J. Plus 135(1), 11 (2020)

71. J.D. Barrow, The area of a rough black hole. arXiv:2004.09444

72. E.N. Saridakis, Barrow holographic dark energy. arXiv:2005.04115

73. E.N. Saridakis, S. Basilakos, The generalized second law of thermodynamics with Barrow entropy. arXiv:2005.08258

74. E.N. Saridakis, Modified cosmology through spacetime thermodynamics and barrow horizon entropy. arXiv:2006.01105

75. D.M. Scolnic et al., The complete light-curve sample of spectroscopically confirmed SNe Ia from Pan-STARRS1 and cosmological constraints from the combined pantheon. Astrophys. J. 859(2), 101 (2018). arXiv: 1710.00845

76. R. Jimenez, A. Loeb, Constraining cosmological parameters based on relative galaxy ages. Astrophys. J. 573, 37-42 (2002). arXiv:astro-ph/0106145

77. M. Moresco, R. Jimenez, L. Verde, L. Pozzetti, A. Cimatti, A. Citro, Setting the stage for cosmic chronometers. I. Assessing the impact of young stellar populations on hubble parameter measurements. Astrophys. J. 868(2), 84 (2018). arXiv: 1804.05864

78. M. Moresco, R. Jimenez, L. Verde, A. Cimatti, L. Pozzetti, Setting the stage for cosmic chronometers. II. Impact of stellar population synthesis models systematics and full covariance matrix. arXiv:2003.07362

79. B.S. Haridasu, V.V. Luković, M. Moresco, N. Vittorio, An improved model-independent assessment of the late-time cosmic expansion. JCAP 10, 015 (2018). arXiv:1805.03595
80. F.K. Anagnostopoulos, S. Basilakos, G. Kofinas, V. Zarikas, Constraining the asymptotically safe cosmology: cosmic acceleration without dark energy. JCAP 02, 053 (2019). arXiv:1806.10580

81. J. Ryan, Y. Chen, B. Ratra, Baryon acoustic oscillation, Hubble parameter, and angular size measurement constraints on the Hubble constant, dark energy dynamics, and spatial curvature. Mon. Not. Roy. Astron. Soc. 488(3), 3844-3856 (2019). arXiv:1902.03196

82. O. Farooq, F.R. Madiyar, S. Crandall, B. Ratra, Hubble parameter measurement constraints on the redshift of the decelerationacceleration transition, dynamical dark energy, and space curvature. Astrophys. J. 835(1), 26 (2017)

83. D. Foreman-Mackey, D.W. Hogg, D. Lang, J. Goodman, emcee: the MCMC hammer Publ. Astron. Soc. Pac. 125, 306 (2013). arXiv: 1202.3665

84. A. Gelman, D.B. Rubin, Inference from iterative simulation using multiple sequences. Stat. Sci. 7, 457 (1992)

85. H. Akaike, A new look at the statistical model identification. IEEE Trans. Autom. Control 19, 716 (1974)

86. G. Schwarz, Estimating the dimension of a model. Ann. Stat. 6(2), 461-464 (1978)

87. D.J. Spiegelhalter, N.G. Best, B.P. Carlin, A.V.D. Linde, Bayesian measures of model complexity and fit. J. R. Stat. Soc. 64(4), 583639 (2002)

88. K. Anderson, Model Selection and Multimodel Inference: A Practical Information-Theoretic Approach, 2nd edn. (Springer, New York, 2002)

89. K.P. Burnham, D.R. Anderson, Multimodel inference: understanding AIC and BIC in model selection. Sociol. Methods Res. 33, 261 (2004)

90. A.R. Liddle, Information criteria for astrophysical model selection. Mon. Not. R. Astron. Soc. 377, L74 (2007). arXiv:astro-ph/0701113

91. F.K. Anagnostopoulos, S. Basilakos, E.N. Saridakis, Bayesian analysis of $f(T)$ gravity using $f \sigma_{8}$ data, Phys. Rev. D 100(8), 083517 (2019). arXiv:1907.07533

92. H. Jeffreys, The theory of probability (Clarendon Press, Oxford, 1998)

93. R.E. Kass, A.E. Raftery, Bayes factors. J. Am. Stat. Assoc. 90(430), 773 (1995)

94. N. Aghanim et al., [Planck Collaboration], Planck 2018 results. VI. Cosmological parameters. arXiv:1807.06209

95. A.G. Riess, S. Casertano, W. Yuan, L.M. Macri, D. Scolnic, Large Magellanic cloud cepheid standards provide a 1 the determination of the hubble constant and stronger evidence for physics beyond $\Lambda$ CDM. Astrophys. J. 876(1), 85 (2019). arXiv:1903.07603 